\title{
Host response to mechanical ventilation for viral respiratory tract infection
}

\author{
Marije P. Hennus*, Riny Janssen", Jeroen L.A. Pennings", Hennie \\ M. Hodemaekers ${ }^{\#}$, Debby Kruijsen ${ }^{\star}$, Nicolaas J. Jansen*, Linde Meyaard ${ }^{+}$, \\ Adrianus J. van Vught* and Louis J. Bont ${ }^{\S}$
}

ABSTRACT: Respiratory syncytial virus (RSV) bronchiolitis causes severe respiratory tract infection in infants, frequently necessitating mechanical ventilatory support. However, life-saving, mechanical ventilation aggravates lung inflammation. We set up a model to dissect the host molecular response to mechanical ventilation in RSV infection. Furthermore, the response to induced hypercapnic acidosis, reported to dampen the inflammatory response to mechanical ventilation in non-infectious models, was assessed.

BALB/c mice were inoculated with RSV or mock-suspension and ventilated for 5 h on day 5 post inoculation.

Mechanical ventilation of infected mice resulted in enhanced cellular influx and increased concentrations of pro-inflammatory cytokines in the bronchoalveolar space. Microarray analysis showed that enhanced inflammation was associated with a molecular signature of a stress response to mechanical ventilation with little effect on the virus-induced innate immune response. Hypercapnic acidosis during mechanical ventilation of infected mice did not change host transcript profiles.

We conclude that mechanical ventilation during RSV infection adds a robust but distinct molecular stress response to virus-induced innate immunity activation, emphasising the importance of lung-protective mechanical ventilation strategies. Induced hypercapnic acidosis has no major effect on host transcription profiles during mechanical ventilation for RSV infection, suggesting that this is a safe approach to minimise ventilator-induced lung injury.

KEYWORDS: Gene expression profiles, hypercapnic acidosis, mice, respiratory syncytial virus, ventilator-induced lung injury

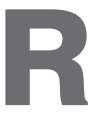
espiratory syncytial virus (RSV) is the most common cause of seasonal acute respiratory tract illness of all ages [1, 2]. More than $50 \%$ of all infants are infected with RSV during the first yr of life and by the age of 2 yrs almost all children have been infected [3]. Of these children $\sim 1-2 \%$ will need hospitalisation and $\sim 10 \%$ of these hospitalised children $(\sim 0.1 \%$ of all children $)$ will require mechanical ventilation for a severe RSV infection during their first yr of life [4]. Hence, RSV infection is the most frequent cause of non-elective paediatric intensive care unit (PICU) admission for mechanical ventilatory support in infants during the winter season [5]. RSV pathogenesis is not completely understood. In humans, severe RSV infection results in a predominantly neutrophil infiltrate in bronchoalveolar lavage fluid (BALF) [6] and a strong innate pro-inflammatory response,

reflected by exhaustion of the peripheral blood neutrophil pool after peak values of viral load and disease severity [7]. In mice, the innate immune response is characterised by induction of type I interferon (IFN)-regulated genes and chemokine genes, and genes involved in inflammation and antigen processing [8].

Although life-saving, mechanical ventilation may induce or aggravate pulmonary inflammation and lung injury. Ventilator-induced lung injury (VILI) results from relatively high tidal volumes $(V T)$, causing alveolar and airway over distension, loss of surfactant and/or mechanical tissue damage $[9,10]$.

No data exists on the consequences of VILI in RSV bronchiolitis patients and tools to study the underlying molecular mechanisms are lacking. Therefore, we designed a murine model in which

This article has supplementary material accessible from www.erj.ersjournals.com Earn CME accreditation by answering questions about this article. You will find these at the back of the printed copy of this journal or online at www.ersjournals.com/misc/cmeinfo.xhtml

\section{AFFILIATIONS}

*Dept of Pediatric Intensive Care, Wilhelmina Children's Hospital, University Medical Center Utrecht, -Dept of Pediatrics, Wilhelmina Children's Hospital, University Medical Center Utrecht,

${ }^{+}$Dept of Immunology, University Medical Center Utrecht, ${ }^{\S}$ Dept of Pediatric Infectious Diseases, Wilhelmina Children's Hospital, University Medical Center Utrecht, Utrecht, and

\# National Institute for Public Health and the Environment, Bilthoven, The Netherlands.

CORRESPONDENCE

M.P. Hennus

Dept of Pediatric Intensive Care, Wilhelmina Children's Hospital University Medical Center Utrecht Lundlaan 6

3584 EA Utrecht

The Netherlands

E-mail: m.p.hennus@umcutrecht.nl

Received:

Oct 122011

Accepted after revision:

March 132012

First published online:

April 102012 
host response to mechanical ventilation in the presence of virusinduced pulmonary inflammation can be studied. In addition, we used the model to study the effects of hypercapnic acidosis on pulmonary inflammation during mechanical ventilation in RSV infection. There is considerable evidence that induced hypercapnic acidosis in noninfectious models may protect against VILI [11-13]. This protective action of hypercapnic acidosis is partly explained by its anti-inflammatory effects reducing the magnitude of host-induced injury: attenuation of neutrophilic oxygen generation and interleukin (IL)-8 production [14], reduction of free radical generation [12] and oxidantinduced tissue damage [11, 14], and the reduction in key cytokines levels, such as tumour necrosis factor (TNF)- $\alpha$ and IL-1 [15]. The effects of hypercapnic acidosis on bacterial injury may vary from benefit to harm depending on the stage of injury process, e.g. depending on whether it is an early or established infection or whether the infection is prolonged [16]. Nothing is known about the effects of hypercapnic acidosis on pulmonary inflammation during mechanical ventilation in viral respiratory tract infection.

We hypothesised that mechanical ventilation enhances the host innate-immune response to RSV. Furthermore, we hypothesised that induced hypercapnic acidosis attenuates VILI in the presence of RSV-induced pulmonary inflammation.

\section{MATERIALS AND METHODS}

The study was approved by the animal care and use committee of the University Medical Center Utrecht (Utrecht, the Netherlands). All animal procedures were carried out in compliance with national and international standards for human care and use of laboratory animals.

\section{Experimental design}

Experiments were performed with male BALB/c mice $(n=60)$ (Harlan, Horst, the Netherlands), aged 6-8 weeks, weighing 21$28 \mathrm{~g}$. On day 0 , mice were randomly assigned to intranasal inoculation with $50 \mu \mathrm{l}$ of either RSV (containing $2 \times 10^{6}$ plaque forming units (pfu) per $50 \mu \mathrm{l}$ ) or mock-suspension. Preparation of virus and mock suspension is described in detail in the online supplementary material. On day 5 , mice were randomly assigned to spontaneous breathing or $5 \mathrm{~h}$ of either normocapnic or hypercapnic mechanical ventilation. This resulted in six experimental groups ( $n=10$ per group): RSV inoculation, spontaneous breathing (RS); RSV-inoculation, normocapnic ventilation (RV); RSV inoculation, hypercapnic ventilation ( $\mathrm{RH})$; Mock inoculation, spontaneous breathing (MS); Mock inoculation, normocapnic ventilation (MV); and mock inoculation, hypercapnic ventilation $(\mathrm{MH})$ (fig. S1, in the online supplementary material). Spontaneously breathing mice were kept in an enclosed environment in which ambient inspiratory oxygen fraction $(\mathrm{FI}, \mathrm{O} 2)$ was maintained at 0.5 for $5 \mathrm{~h}$ with and without $5 \%$ $\mathrm{CO}_{2}$. Mice assigned to mechanical ventilation $\left(V \mathrm{~T} 12 \mathrm{~mL} \cdot \mathrm{kg}^{-1}\right.$, frequency $40 \cdot \mathrm{min}^{-1}$, positive end-expiratory pressure (PEEP) $6 \mathrm{cmH}_{2} \mathrm{O}$ and $\mathrm{FI}_{2} \mathrm{O}_{2} 0.5$ ), were anesthetised, ventilated and monitored for $5 \mathrm{~h}$, as described in the online supplementary material. We used a high $V \mathrm{~T}$ in order to provoke lung injury, enabling us to study the effects of hypercapnic acidosis on VILI. Hypercapnic acidosis during mechanical ventilation was induced by adding $5 \% \mathrm{CO}_{2}$ to the inspired gas mixture while maintaining the $\mathrm{II}_{1} \mathrm{O}_{2}$ at 0.5 . The inspired gas mixture was monitored continuously during the whole experiment (Datex Cardiocap Monitor, GE Healthcare, Chalfont St Giles, UK). Systolic blood pressure and heart rate of ventilated mice were monitored non-invasively using a murine tail-cuff system (ADInstruments $\mathrm{GmbH}$, Spechbach Germany). All mice survived 5 h of mechanical ventilation and remained haemodynamically and respiratory stable throughout the whole experiment (table 1).

\section{Measurements}

After exsanguination, BALF of six mice per experimental group were collected for total and differential cell counts as described in the online supplementary material. TNF- $\alpha$, IL- 6 and keratinocytederived chemokine $(\mathrm{KC})$ concentrations in BALF supernatant were measured by ELISA in accordance with the manufacturer's instructions (R\&D Systems, Abingdon, UK). Pulmonary RSV-A concentrations were analysed by real-time PCR as described previously $[8,17]$.

The lungs of four mice per experimental group were used for microarray analysis as described in detail in the online supplementary material. Gene expression changes were visualised using a heat map and principal component analysis. Principal component analysis is a mathematical algorithm that describes data on the basis of their (dis)similarity, so that greater distances correspond to greater dissimilarity [18]. In this analysis, a principal component is defined as a mathematically derived combination of genes and their expression characteristics that can be used to describe part of the process observed.

\begin{tabular}{|c|c|c|c|c|}
\hline \multirow[t]{3}{*}{ TABLE 1} & \multicolumn{4}{|c|}{$\begin{array}{l}\text { Haemodynamic and respiratory characteristics } \\
\text { of ventilated mice }\end{array}$} \\
\hline & \multicolumn{4}{|c|}{ Ventilated mice } \\
\hline & MV & MH & RV & RH \\
\hline \multicolumn{5}{|l|}{ HR beats $\mathrm{min}^{-1}$} \\
\hline $\mathrm{Oh}$ & $403 \pm 45$ & $374 \pm 33$ & $427 \pm 40$ & $420 \pm 38$ \\
\hline $2.5 \mathrm{~h}$ & $393 \pm 37$ & $432 \pm 66$ & $452 \pm 28$ & $480 \pm 40$ \\
\hline $5 \mathrm{~h}$ & $362 \pm 28$ & $413 \pm 78$ & $441 \pm 27$ & $478 \pm 39$ \\
\hline \multicolumn{5}{|l|}{$\mathrm{BP} \mathrm{mmHg}$} \\
\hline $\mathrm{Oh}$ & $91 \pm 16$ & $88 \pm 9$ & $93 \pm 12$ & $96 \pm 15$ \\
\hline $2.5 \mathrm{~h}$ & $91 \pm 13$ & $93 \pm 9$ & $101 \pm 19$ & $100 \pm 22$ \\
\hline $5 \mathrm{~h}$ & $95 \pm 15$ & $98 \pm 21$ & $98 \pm 14$ & $104 \pm 15$ \\
\hline pH & $7.41 \pm 0.03$ & $7.22 \pm 0,05^{\star}$ & $7.40 \pm 0,05$ & $7.18 \pm 0.08^{\#}$ \\
\hline $\mathrm{Pa}, \mathrm{CO}_{2} \mathrm{kPa}$ & $42.9 \pm 3$ & $78.5 \pm 9^{\star}$ & $37.9 \pm 5$ & $71.5 \pm 4^{\#}$ \\
\hline $\mathrm{Pa}_{\mathrm{a}} \mathrm{O}_{2} \mathrm{KPa}$ & $272 \pm 13$ & $274 \pm 14$ & $254 \pm 7$ & $248 \pm 16$ \\
\hline $\mathrm{BE} \mathrm{mmol} \cdot \mathrm{L}^{-1}$ & $3 \pm 3$ & $4 \pm 4$ & $-1 \pm 2$ & $-1 \pm 6$ \\
\hline $\mathrm{HCO}_{3}^{-} \mathrm{mmol} \cdot \mathrm{L}^{-1}$ & $27.6 \pm 2$ & $31.8 \pm 3$ & $23.3 \pm 2$ & $30.4 \pm 4$ \\
\hline \multicolumn{5}{|c|}{$\begin{array}{l}\text { Data are presented as mean } \pm \mathrm{SD} \text {. MV: mock inoculation, normocapnic } \\
\text { mechanical ventilation; } \mathrm{MH} \text { : mock inoculation, hypercapnic mechanical } \\
\text { ventilation; RV: respiratory syncytial virus (RSV) inoculation, normocapnic } \\
\text { mechanical ventilation; } \mathrm{RH} \text { : RSV inoculation, hypercapnic mechanical ventila- } \\
\text { tion; } \mathrm{HR} \text { : heart rate: } \mathrm{BP} \text { : systolic blood pressure; } \mathrm{Pa}_{2} \mathrm{CO}_{2} \text { : arterial carbon dioxide } \\
\text { tension; } \mathrm{Pa}, \mathrm{O}_{2} \text { arterial oxygen tension; } \mathrm{BE}: \text { and } \mathrm{HCO}_{3}^{-} \text {in }{ }^{*} \mathrm{p}<0.05 \text { compared } \\
\text { with } \mathrm{MV} \text { controls using paired t-test. } \#: \mathrm{p}<0.05 \text { compared with } \mathrm{RV} \text { controls } \\
\text { using paired t-test. }\end{array}$} \\
\hline
\end{tabular}


Differentially regulated genes were subdivided into 12 functional classes or pathways based on their function as described before [17]. Pathway enrichment was used to determine which pathways were involved in responses found for pair-wise group comparisons.

\section{Statistical analysis}

All data in the results are expressed as mean \pm SD. A 2-way ANOVA was used to compare the means of multiple groups; allowing for interaction between virus and mechanical ventilation. For paired data (table 1), unpaired t-test was used. A pvalue $<0.05$ was considered statistically significant. Statistical analyses were carried out using GraphPad PRISM 5 (La Jolla, San Diego, CA, USA).

Microarray analysis was performed using $\mathrm{R}$ version 2.13 .1 ( $\mathrm{R}$ Development Core Team; www.r-project.org). Regulated genes were identified with ANOVA using $\mathrm{p}<0.001$ (false discovery rate $<5 \%$ ) and fold ratio $>2$ as significance criteria. Data are presented as means for four mice per group.

\section{RESULTS}

\section{Mechanical ventilation during RSV infection enhances pulmonary inflammation}

Compared with spontaneous breathing MS mice, mechanical ventilation induced an influx of BALF total cell count, neutrophils and macrophages (fig. 1a) and increased BALF IL-6 and KC concentrations (fig. 1b). Compared with the mock inoculation (MS mice), RSV infection in the spontaneous breathing mice (RS) induced a mixed-cell influx into the bronchoalveolar space fig. 1a) and elevated levels of IL-6 and $\mathrm{KC}$ (fig. 1b). Mechanical ventilation resulted in a robust inflammatory response with enhanced influx of BALF cells (fig 1a). In addition a significant increase in IL-6 and KC was observed in the RV mice when compared with the RS mice (fig. 1b). No differences in viral loads were observed between ventilated and spontaneous breathing mice (data not shown). In conclusion mechanical ventilation during RSV infection enhanced pulmonary inflammation, reflected by BALF-cellular responses and pro-inflammatory cytokines, when compared with either mechanical ventilation or RSV infection alone.

\section{RSV induced inflammation and VILI are characterised by distinct host-transcription profiles}

Microarray analysis showed a total of 793 genes to be differentially regulated in this study. When comparing spontaneous breathing mock-inoculated mice (MS) to ventilated mockinoculated mice (MV), 376 genes were differentially regulated (fig. 2); 265 upregulated genes and 111 downregulated genes (table 2). Functional enrichment analysis showed that the stressresponse and acute inflammatory-response pathways were enriched among differentially upregulated genes (table 3). A total of 408 genes were differentially regulated when comparing spontaneous breathing mock-inoculated mice (MS) with RSVinoculated mice (RV) (fig. 2); 382 upregulated genes and 26 downregulated genes (table 2). As we have described previously [17], primary RSV infection induced a robust innate immune response characterized by differential expression of chemokines, interferon responses and antigen processing pathways (table 3).
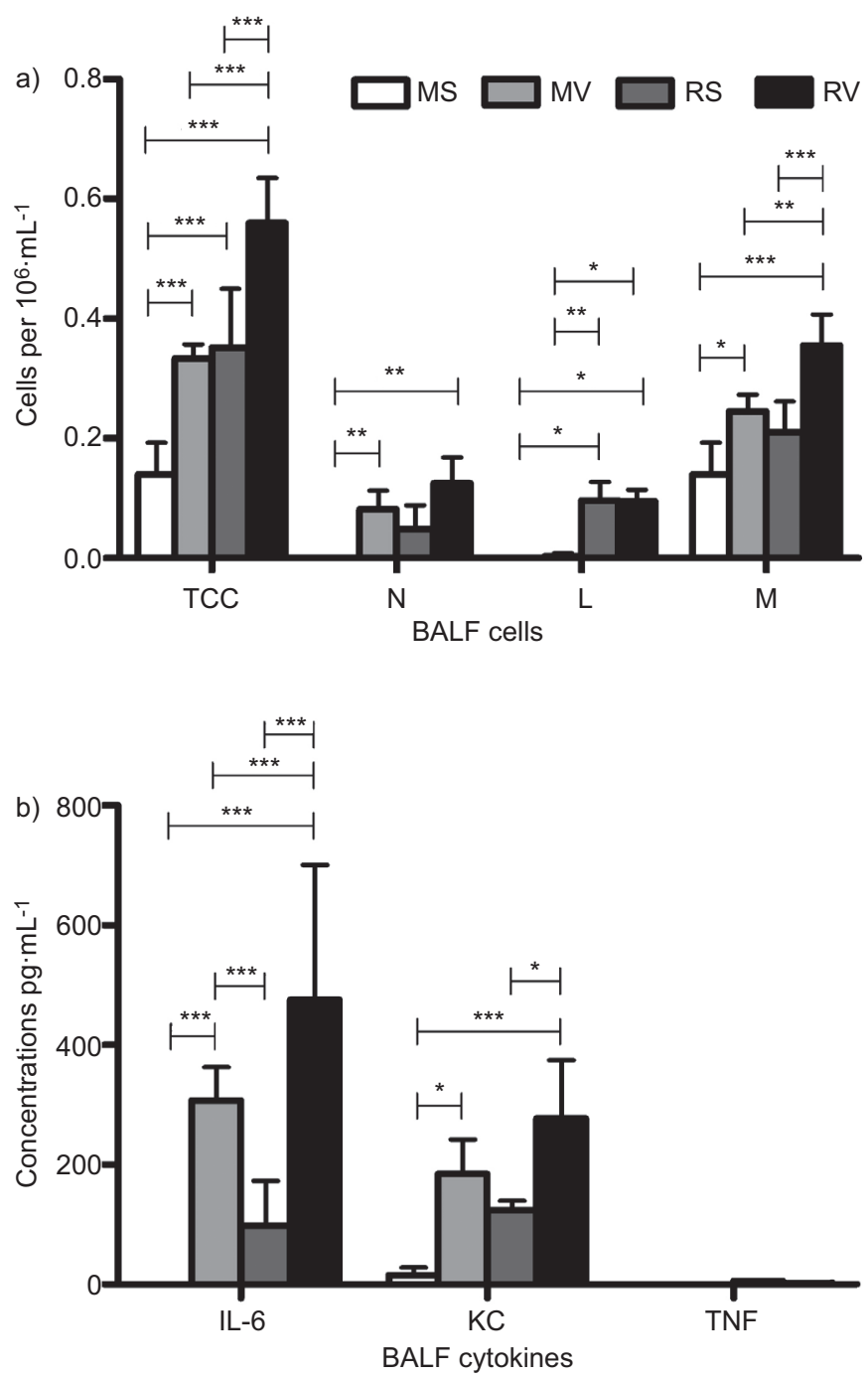

FIGURE 1. Bronchoalveolar lavage fluid (BALF) cellular responses and cytokine concentrations on day 5 ( $n=6$ for each group). a) Data showing mean \pm SD of BALF pulmonary cellular responses. b) Data showing mean $\pm S D$ of BALF cytokines. BALB/c mice were inoculated with respiratory syncytial virus (RSV; $2 \times 10^{6}$ plaque forming units) or mock suspension on day 0 and subjected to $5 \mathrm{~h}$ of mechanical ventilation on day 5. MS: mock inoculation, spontaneous breathing; MV: mock inoculation, mechanical ventilation; RS: RSV inoculation, spontaneous breathing; RV: RSV inoculation, mechanical ventilation; TCC: total cell count; N: neutrophils; L: leukocytes; M: macrophages; IL-6: interleukin-6; KC: keratinocytederived chemokine; TNF: tumour necrosis factor. Statistical analysis used two-way ANOVA. *: $p<0.05 ; * *: p<0.01 ; * * *: p<0.001$.

Mechanical ventilation of RSV-inoculated mice (RV) induced a gene expression profile response distinct from mice profiles in spontaneous breathing RSV-inoculated mice (RS) or mechanically ventilated mock-inoculated mice (MV) (fig. 3). This was accented by the principal-component analysis (fig. 4). Clusters of spontaneous breathing, ventilated, mock- or RSV-inoculated mice can be clearly distinguished. This analysis indicated that the responses to RSV infection and to mechanical ventilation are strong, but distinct. A total of 293 genes were differentially regulated when comparing spontaneous breathing RSV-inoculated mice (RS) with ventilated RSV-inoculated mice (RV) 


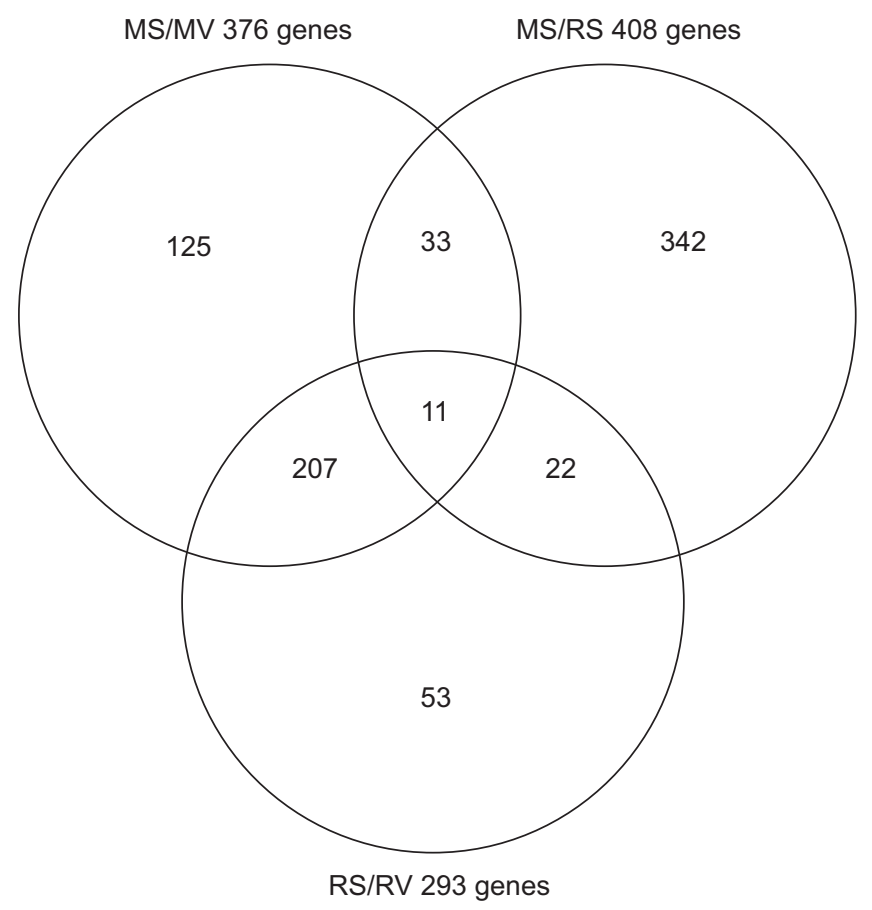

FIGURE 2. Number of genes differentially regulated in three group comparisons. A total of 793 genes were differentially regulated with a greater than two-fold change ( $n=4$ for each group; $p<0.001$ ) in lungs of respiratory syncytial virus (RSV)or mock-inoculated mice on day 5 after either $5 \mathrm{~h}$ of mechanical ventilation or spontaneous breathing. The numbers in the cross sections of the circles reflect the number of overlapping differentially regulated genes in the three group comparisons. MS: mock inoculation, spontaneous breathing; MV: mock inoculation, normocapnic mechanical ventilation, RS: RSV spontaneous breathing, RV: RSV inoculated, normocapnic mechanical ventilation. MS/MV: ventilation effect; MS/RS: RSV effect; RS/RV: effect of mechanical ventilation during RSV infection.

(fig. 2); 227 upregulated and 66 downregulated genes (table 2). The stress-response and acute-inflammatory-response pathway were enriched (table 3). Of all 293 differentially regulated genes in ventilated RSV-inoculated mice, 218 (74\%) genes were also regulated in ventilated mock-inoculated mice (fig. 2). All 218 genes were concordantly (174 versus $174 \mathrm{up} / \mathrm{up}$ and 44 versus 44 down/down) differentially regulated (data not shown), suggesting a robust ventilation effect additive to the RSV-induced response.

Mechanical ventilation had a modest effect on the expression of genes induced by RSV infection. Mechanical ventilation regulated the expression of $33 \mathrm{RSV}$-induced genes, of which 27 were discordant (fig. 2 and fig. 4). A partial downregulation was observed in the chemokine pathway (table 3) and in TNF- $\alpha$ and IFN- $\gamma$ expression. It is noted that the chemokines TNF- $\alpha$ and IFN- $\gamma$ expression in ventilated RSV-inoculated mice (RV) was still strongly upregulated when compared with mock-inoculated spontaneous breathing mice (MS) (table 4).

53 genes were only differentially regulated in ventilated RSVinoculated mice (fig. 2); these genes were not regulated by either simple RSV infection or mechanical ventilation. Of these genes, none belonged to the innate immune response and only six to the stress response: RIKEN cDNA 2310016C08 gene (2310016C08RIK), adrenergic receptor beta-2 (ADRB2), cathelicidin antimicrobial

\begin{tabular}{|c|c|c|c|c|c|c|}
\hline $\begin{array}{ll}\text { ABLE } 2 & \text { Number } \\
& \text { pathway }\end{array}$ & I & $\begin{array}{l}\text { ntial re } \\
\text { y grou }\end{array}$ & 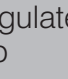 & ge & & \\
\hline \multirow[t]{2}{*}{ Pathways } & \multicolumn{2}{|c|}{ MS/MV } & \multicolumn{2}{|c|}{ MS/RS } & \multicolumn{2}{|c|}{ RS/RV } \\
\hline & Up & Down & Up & Down & Up & Down \\
\hline $\begin{array}{l}\text { Acute inflammatory } \\
\text { response }\end{array}$ & 10 & 0 & 17 & 0 & 4 & 0 \\
\hline Chemokine & 1 & 1 & 19 & 0 & 1 & 8 \\
\hline Antigen processing & 0 & 2 & 28 & 0 & 0 & 1 \\
\hline Interferon signalling & 4 & 2 & 39 & 0 & 3 & 4 \\
\hline Inflammatory response & 8 & 1 & 23 & 0 & 5 & 2 \\
\hline Immune response & 9 & 6 & 32 & 0 & 6 & 3 \\
\hline Stress response & 29 & 4 & 9 & 2 & 30 & 2 \\
\hline Apoptosis & 10 & 1 & 12 & 0 & 6 & 0 \\
\hline Cell cycle & 10 & 1 & 28 & 0 & 7 & 0 \\
\hline Metabolism & 58 & 28 & 45 & 7 & 45 & 19 \\
\hline Various function & 70 & 51 & 107 & 17 & 63 & 19 \\
\hline Unknown & 60 & 14 & 23 & 0 & 57 & 8 \\
\hline $\begin{array}{l}\text { Differentially regulated/ } \\
\text { group }\end{array}$ & 265 & 111 & 382 & 26 & 227 & 66 \\
\hline Total & & 376 & & 408 & & 293 \\
\hline \multicolumn{7}{|c|}{$\begin{array}{l}\mathrm{n}=4 \text { for each group. Based on their function, genes were subdivided into } 12 \\
\text { more specific immunological (and other) pathways [17]. The number of genes } \\
\text { known was taken from Gene Ontology, in which one gene can be placed in } \\
\text { multiple pathways. A number of upregulated (Up) and downregulated (Down) } \\
\text { genes were distinguished. MS: mock inoculation, spontaneous breathing; MV: } \\
\text { mock inoculation, normocapnic mechanical ventilation; RS: respiratory syncy- } \\
\text { tial virus (RSV) inoculation, spontaneous breathing; RV: RSV inoculation, } \\
\text { normocapnic mechanical ventilation. MS/MV: ventilation effect; MS/RS: RSV } \\
\text { effect, RS/RV: effect of mechanical ventilation during RSV infection. }\end{array}$} \\
\hline
\end{tabular}

peptide $(C A M P)$, coagulation factor XIII A1 subunit (F13A1), neutrophilic granule protein (NGP), and tyrosine aminotransferase (TAT). 51 (96\%) genes had fold expression levels near cut-off values (two fold) suggesting minimal effects. Only NGP and Prokineticin 2 (PROK2) were >3-fold upregulated (supplementary table S1). In conclusion gene expression analysis demonstrates that the enhanced inflammatory response observed in ventilated RSV infected mice is predominantly attributable to the molecular stress response to mechanical ventilation.

\section{Response to induced hypercapnic mechanical-ventilation for RSV infection}

In mock-inoculated mice, hypercapnic acidosis during mechanical ventilation $(\mathrm{MH})$ did not influence the host response as expressed by BALF cellular responses, cytokine concentrations (online supplementary material fig. S2) or differential expression of any gene (fig. 3 and 5). Similarly, hypercapnic ventilation of RSV-inoculated mice (RH) did not affect BALF cellular responses, cytokine concentrations (online supplementary material fig. S2) and did not result in differential expression of any gene (figs 3 and 5). No differences in viral loads were observed between mice after $5 \mathrm{~h}$ of normocapnic or hypercapnic ventilation (data not shown).

\section{DISCUSSION}

We investigated the effects of mechanical ventilation as well as hypercapnic acidosis during mechanical ventilation on the host 


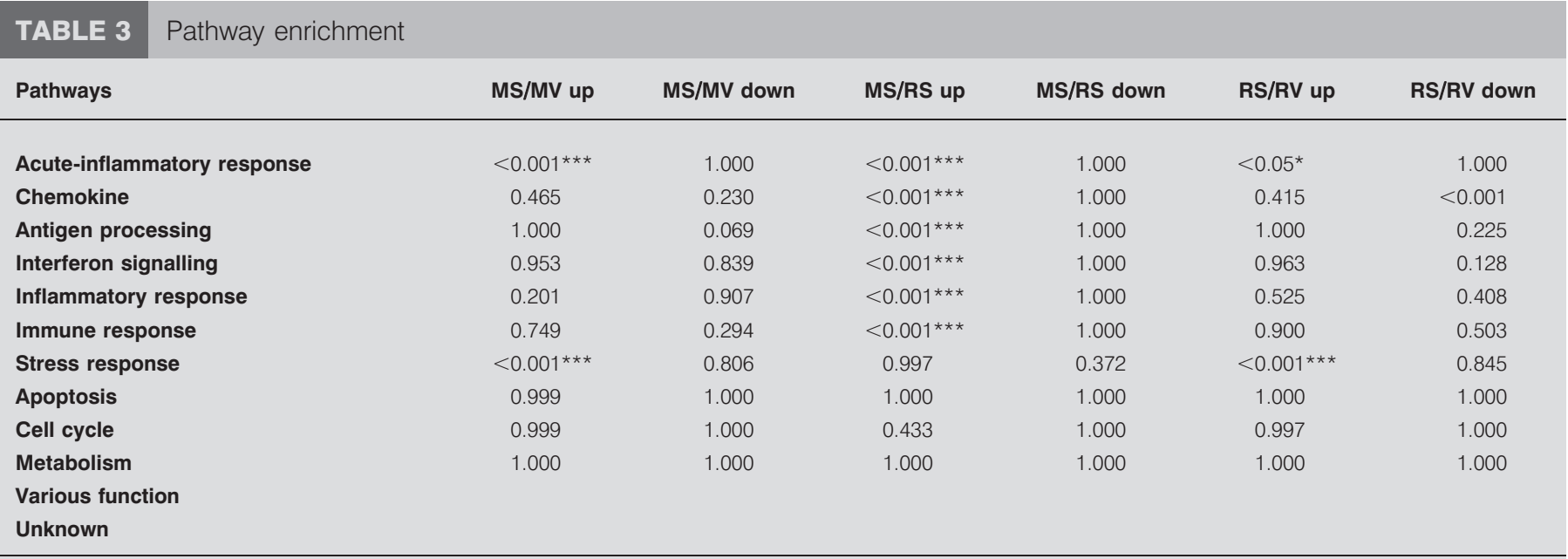

Using data from table 2, a binomial test was applied to determine in which pathways significantly more genes were present in the list of upregulated/downregulated genes for the three group comparisons than could be expected by chance. MS: mock inoculation, spontaneous breathing; MV: mock inoculation, normocapnic mechanical ventilation; RS: respiratory syncytial virus (RSV) inoculation, spontaneous breathing; RV: RSV inoculation, normocapnic mechanical ventilation; MS/MV ventilation effect; MS/RS: RSV effect; RS/RV: effect of mechanical ventilation during RSV infection. Using binomial test: *: $p<0.05 ;{ }^{* * *}: p<0.001$.

response during RSV-infection. We found that mechanical ventilation provoked a robust effect characterised by distinct molecular stress- and acute-inflammatory responses additive to, but not aggravating, the innate immune response seen in RSV. Furthermore, hypercapnic ventilation of RSV-infected mice did

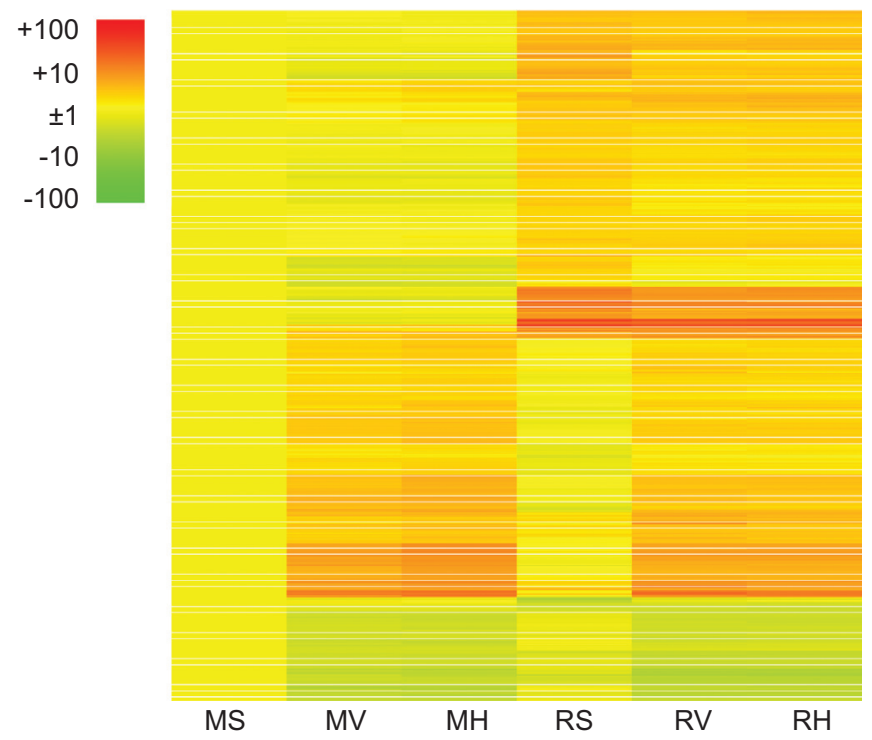

FIGURE 3. Cluster analysis of regulated genes in lungs of respiratory syncytial virus- (RSV) or mock-inoculated mice on day 5 after either $5 \mathrm{~h}$ of mechanical ventilation or spontaneous breathing ( $\mathrm{n}=4$ for each group). All genes with $\mathrm{a}>2.0$ fold change $(p<0.001$ via ANOVA) are depicted. Gene expression changes are shown relatively to the median for spontaneous breathing mock-inoculated mice. Each row represents lungs of a group of mice. MS: mock inoculation, spontaneous breathing; MV: mock inoculation, normocapnic mechanical ventilation; MH: mock inoculation, hypercapnic mechanical ventilation; RS: respiratory syncytial virus (RSV) inoculation, spontaneous breathing; RV: RSV inoculation, normocapnic mechanical ventilation, RH: RSV inoculation, hypercapnic mechanical ventilation. not modulate pulmonary inflammation expressed by BALF cellular responses, cytokine concentrations or gene expression profiles.

The results from our experiments, in which only mechanical ventilation or RSV-infection was applied, corroborated with existing literature. Mechanical ventilation of mock-inoculated mice significantly enhanced both pulmonary cellular responses and cytokine concentrations $[9,19]$. Gene transcription profiles during mechanical ventilation, known to produce a global transcriptional signature distinct from spontaneous breathing, were similar to those reported. GHARIB et al. [20] demonstrated that $4 \mathrm{~h}$ of mechanical ventilation $\left(V \mathrm{~T} 10 \mathrm{~mL} \cdot \mathrm{g}^{-1}\right)$ activated a pro-inflammatory transcriptional programme in lungs of C57BL/ 6 mice, reflected by enrichment of 176 genes in proinflammatory pathways. Of these 176 genes, 57 were found in our study with 93\% concordant effects (upregulation versus downregulation). PAPAIAHGARI et al. [21] studied gene expression in CD-1/ICR mice ventilated for $2 \mathrm{~h}\left(V \mathrm{~T} 12 \mathrm{~mL} \cdot \mathrm{kg}^{-1}\right)$. They found 63 differentially regulated genes of which 31 were differentially regulated in our study with $97 \%$ concordant effects. AltEMEIER et al. [22] showed modest differential gene expression in $\mathrm{C} 57 \mathrm{Bl} / 6$ mice after $4 \mathrm{~h}$ of mechanical ventilation with a lower $V \mathrm{~T}\left(10 \mathrm{~mL} \cdot \mathrm{kg}^{-1}\right)$. Of 32 differentially regulated genes found in their study, 16 were found in our study with $94 \%$ concordant effects. Although overlapping genes in these studies predominantly behaved concordantly, we found a larger number of 376 differentially regulated genes. We hypothesised that this was caused by differences in duration of mechanical ventilation, ventilator settings and mouse species.

RSV infection of BALB/c mice represents a well-established experimental model, which has successfully been used to study the immunopathogenesis of RSV [23-25]. Lymphocytic response on day 5 post-infection and elevated levels of TNF- $\alpha$, IL-6, KC and viral loads characterised RSV-infection in our study, consist with literature findings. Transcription profiles of primary RSV-response in BALB/c mice on day 5 after infection 


Gene
ACAA1B
AIF1
ALAS2
CCL12
CCL2
CCL4
CCL7
CD52
CH25H
CLEC12A
CSRNP1
CXCL10
CXCL11
CXCL12
CXCL2
EOMES
HBA-A1
HBB-B1
HBB-B2
IFIT2
IFIT3
IFNG
LTB
MT2
MX1
PHF11
SCGB1A1
SERPINA3N
SNCA
THBS1
TNF
XCL1
ZBTB16

\begin{tabular}{|c|c|c|c|}
\hline MN/MS & RS/MS & RN/MS & RN/RS \\
\hline 1.80 & 0.27 & 0.66 & 2.46 \\
\hline 0.60 & 5.30 & 2.55 & 0.48 \\
\hline 0.93 & 0.35 & 0.77 & 2.20 \\
\hline 0.74 & 4.88 & 1.77 & 0.36 \\
0.95 & 32.51 & 8.34 & 0.26 \\
0.99 & 5.50 & 2.51 & 0.46 \\
0.92 & 7.68 & 2.28 & 0.30 \\
\hline 0.42 & 2.76 & 1.31 & 0.47 \\
\hline 1.72 & 3.67 & 1.75 & 0.48 \\
\hline 0.46 & 2.42 & 0.98 & 0.41 \\
\hline 4.42 & 2.05 & 5.50 & 2.69 \\
\hline 0.98 & 36.28 & 13.59 & 0.37 \\
0.99 & 6.32 & 3.01 & 0.48 \\
\hline 0.98 & 3.30 & 1.56 & 0.47 \\
\hline 3.84 & 2.23 & 5.54 & 2.49 \\
\hline 0.50 & 2.33 & 1.06 & 0.46 \\
\hline 0.72 & 0.20 & 0.56 & 2.74 \\
\hline 0.79 & 0.21 & 0.65 & 3.09 \\
\hline 0.88 & 0,20 & 0.63 & 3.21 \\
\hline 0.53 & 4.76 & 1.97 & 0.41 \\
\hline 0.44 & 3.15 & 1.54 & 0.49 \\
\hline 0.97 & 5.73 & 2.34 & 0.41 \\
\hline 0.48 & 2.03 & 0.94 & 0.47 \\
\hline 11.51 & 2.85 & 13.07 & 4.59 \\
\hline 0.78 & 5.60 & 2.62 & 0.47 \\
\hline 0.69 & 4.08 & 2.01 & 0.49 \\
\hline 0.35 & 0.45 & 0.21 & 0.47 \\
\hline 6.11 & 2.27 & 7.72 & 3.39 \\
\hline 0.90 & 0.27 & 0.79 & 2.95 \\
\hline 5.95 & 2.97 & 7.14 & 2.41 \\
\hline 0.82 & 2.94 & 1.27 & 0.43 \\
\hline 0.80 & 4.41 & 1.84 & 0.42 \\
\hline 4.18 & 0.43 & 2.72 & 6.38 \\
\hline
\end{tabular}

\author{
Gene name \\ Acetyl-coenzyme A acyltransferase 1B \\ Allograft inflammatory factor 1 \\ Aminolevulinic acid synthase 2, erythroid \\ Chemokine (C-C motif) ligand 12 \\ Chemokine (C-C motif) ligand 2 \\ Chemokine (C-C motif) ligand 4 \\ Chemokine (C-C motif) ligand 7 \\ CD52 antigen \\ Cholesterol 25-hydroxylase \\ C-type lectin domain family 12 , member a \\ Cysteine-serine-rich nuclear protein 1 \\ Chemokine (C-X-C motif) ligand 10 \\ Chemokine (C-X-C motif) ligand 11 \\ Chemokine (C-X-C motif) ligand 12 \\ Chemokine ( $\mathrm{C}-\mathrm{X}-\mathrm{C}$ motif) ligand 2 \\ Eomesodermin homologue (Xenopus laevis) \\ Haemoglobin- $\alpha$ adult chain 1 \\ Haemoglobin- $\beta$ adult major chain \\ Haemoglobin- $\beta$ adult minor chain \\ Interferon-induced protein with tetratricopeptide repeats 2 \\ Interferon-induced protein with tetratricopeptide repeats 3 \\ Interferon- $\gamma$ \\ Lymphotoxin B \\ Metallothionein 2 \\ Myxovirus (influenza virus) resistance 1 \\ PHD finger protein 11 \\ Secretoglobin, family $1 \mathrm{~A}$, member 1 (uteroglobin) \\ Serine (or cysteine) peptidase inhibitor, clade A, member $3 \mathrm{~N}$ \\ Synuclein- $\alpha$ \\ Thrombospondin 1 \\ Tumour necrosis factor \\ Chemokine ( $\mathrm{C}$ motif) ligand 1 \\ Zinc finger and BTB domain containing 16
}

FIGURE 4. Differentially regulated genes in both mock-inoculated, spontaneous breathing (MS) mice/respiratory syncytial virus (RSV)-inoculated, spontaneously breathing (RS) mice and RS mice/RSV-inoculated normocapnic-ventilated (RV) mice. When MS/RS were mice were compared with RS/RV mice, 33 genes were found to be differentially regulated in both groups, mostly chemokines. The modest downregulation of chemokine expression in ventilated RSV-inoculated mice (RS/RV) was still strongly upregulated when compared with spontaneous breathing mock-inoculated animals (MS/RV). Red box: greater than two-fold change in upregulation; green box: greater than two-fold change in downregulation; MV: mock inoculation, normocapnic mechanical ventilation; MS/MV: ventilation effect, MS/RS: RSV-effect; RS/RV: the effect of mechanical ventilation during RSV infection.

have been reported [8, 17]. We previously found 188 differentially regulated genes [17], of which 137 (73\%) were also differentially regulated in our study with $100 \%$ concordant effects. As the responses of our VILI and RSV models were in line with findings published earlier, our model provided a suitable framework for studies on mechanical ventilation during RSV infection.

Little data exists on the effect of mechanical ventilation on pulmonary inflammation in the presence of a viral infection. BEM et al. [26] showed that infection with mechanical ventilation enhanced the response to pneumonia virus of mice (PVM) and might serve to exacerbate local inflammatory responses and lung injury. However, ZOSKY et al. [27] showed that mechanical ventilation did not further exacerbate lung function impairment or lung inflammation following influenza A infection, using the same $V$ T and mice species. No data exists on the consequences of VILI on RSV-induced pulmonary inflammation despite the fact that RSV infection is the most frequent cause of non-elective PICU admission for mechanical ventilatory support in infants during the winter season.
We showed that mechanical ventilation during RSV infection added a stress response to virus-induced innate immunity activation with only little interaction, reflected by modest inhibition of TNF- $\alpha$ and IFN- $\gamma$ chemokine expression pattern. We hypothesise that the additional stress response may cause further damage and inhibition of innate immune response with subsequent delayed viral clearance. Although one should be cautious in translating results from experimental models to daily clinical practice, the observed ventilation-specific lung inflammation in addition to the response to viral respiratory infections underscores the need for optimal modes of lung protective ventilation in patients with severe respiratory infections. More specifically, these results support the notion to reconsider invasive mechanical ventilation for RSV bronchiolitis in favour of less invasive mechanical ventilation strategies.

Of the 53 genes only differentially regulated in ventilated RSVinoculated mice, $96 \%$ had fold expression levels near cut-off values (two fold) suggesting minimal effects. Only neutrophilic NGP and PROK2 were upregulated more than three fold. NGP was first identified as a myeloid-specific granule protein with 


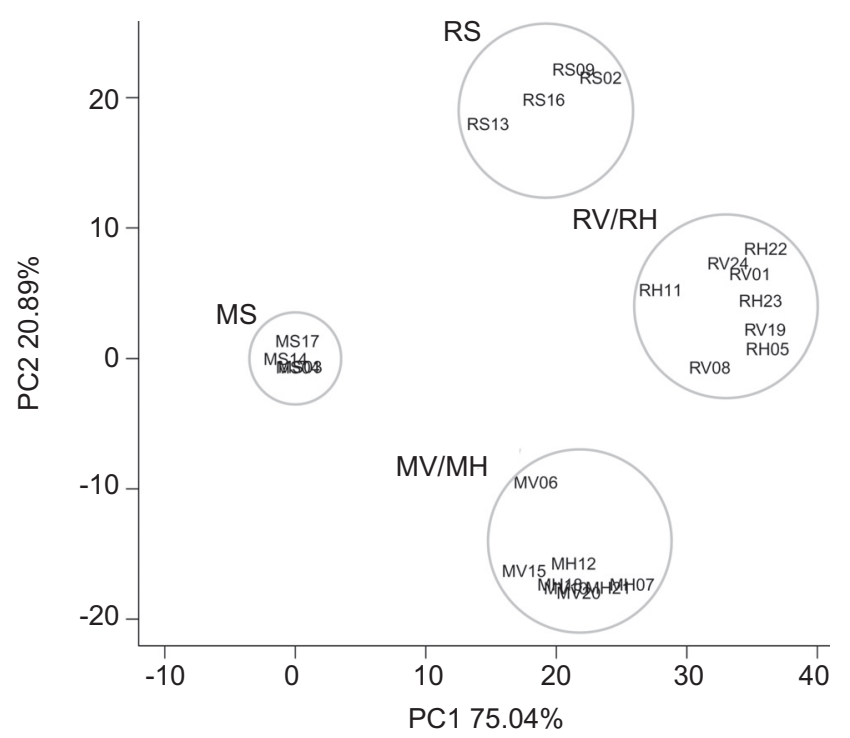

FIGURE 5. Principal-component analysis (PCA) of the microarray analysis of the BALB/c mice lungs ( $n=4$ for each group). PCA identifies the two directions (PC1 and PC2) along which data have the largest spread. The distance between the groups in the figure corresponds to the combined differences in gene expression in the groups. Every letter combination above a circle (e.g. RS or MS) corresponds to one of the six experimental groups ( $n=4$ mice/group). Each letter/number combination inside a circle corresponds to a single mouse. (e.g. $\mathrm{RH} 11=$ respiratory syncytial virus $(R)$, hypercapnic mechanical ventilation $(H)$, mouse number 11$)$. MS: mock inoculation, spontaneous breathing; MV: mock inoculation, normocapnic mechanical ventilation; $\mathrm{MH}$ : mock inoculation, hypercapnic mechanical ventilation; RS: respiratory syncytial virus (RSV) inoculation, spontaneous breathing; RV: RSV inoculation, normocapnic mechanical ventilation, RH: RSV inoculation, hypercapnic mechanical ventilation.

homology to cystatin superfamily [28], known to modulate the activity of the immune response, but has no known human homologue. PROK2 belongs to a family of secreted peptides with diverse regulatory roles, e.g. modulation of immunity [29]. The importance of both genes in mechanical ventilation for RSV infection remains to be elucidated.

Studies have shown that induced hypercapnic acidosis in noninfectious models may protect against VILI [11, 12]. The effects of hypercapnic acidosis on bacterial injury may vary from benefit to harm depending on the stage of injury process [16]. We have found that induced hypercapnic acidosis during mechanical ventilation did not influence pulmonary inflammation expressed by BALF cellular responses and cytokine concentrations in either our noninfectious or infectious model. These findings were supported by the complete absence of induction or suppression of gene expression. Because $V \mathrm{~T}$ and ventilator settings were maintained constant throughout the experiment, results are solely attributable to the effects of hypercapnic acidosis. However, we cannot exclude deleterious effects of either higher concentrations of carbon dioxide or hypercapnic acidosis during prolonged mechanical ventilation for viral infection. The clinically relevant conclusion drawn from our study is that hypercapnic acidosis, frequently accepted during the commonly applied low $V \mathrm{~T}$ and limited pressure ventilation strategies in RSV-infected infants requiring respiratory support
[30], does not aggravate VILI against the background of virusinduced airway inflammation.

This study has several strengths. First, we provide a novel animal model in which the molecular effects of stable mechanical ventilation on RSV lower-respiratory tract infection can be studied. Secondly, this model enables studying the molecular effects of several interventions aimed at reducing pulmonary inflammation such as ventilator strategies, immunosuppression and relative hypoxia. Thirdly, knowledge of the effects of hypercapnic acidosis during mechanical ventilation during RSV infection is of clinical importance. We provided evidence that hypercapnic acidosis, often the resultant of lung protective ventilator strategies, did not alter viral replication or host response during mechanical ventilation for RSV infection in a mouse model. However, we have also considered the following limitations: Firstly RSV is not a natural murine pathogen; nonetheless, the mouse model shows similarity to the pathogenesis of RSV-induced lower airway disease in humans [25]. An alternative mouse model used to mimic human RSV infection is PVM, a natural rodent pathogen, belonging to the same virus family, subfamily and genus as RSV [31,32]. However, PVM does not elicit the characteristic lymphocytic response as seen in both human and murine RSV infection on day 4-6 after infection [33]; with T-cells having an important role in both viral clearance and disease enhancement in mice [24]. Secondly, the time course of mechanical ventilation in this study is short whereas children with a respiratory insufficiency due to a RSV infection usually require days, even weeks, of mechanical ventilation. Our conclusion that VILI does not affect the innate response to viral infection needs to be tested further in a model exposing the mice to longer ventilation. Thirdly, in our experimental setting it was not possible to measure heart rate and blood pressure in nonsedated mice. In addition, we hypothesise that non-sedated mice may have had less severe induced hypercapnic acidosis, due to increased rate of breathing in response to $5 \% \mathrm{CO}_{2}$ air. Finally, we used transcription profiling of whole lungs to obtain expression values and therefore have not determined the cell-specific source of differentially expressed genes.

In summary, we have developed a model to study the molecular mechanisms of mechanical ventilation during viral infection. This study advances our knowledge of to what extent mechanical ventilation affects pulmonary inflammation in RSV infection. We demonstrate that mechanical ventilation adds a stress response to virus-induced innate immunity activation. Furthermore, our model suggests that hypercapnic acidosis, frequently accepted during mechanical ventilation of RSV-infected infants, does not aggravate VILI or affect the local immune response against the background of virus-induced airway inflammation.

\section{STATEMENT OF INTEREST}

None declared.

\section{ACKNOWLEDGEMENTS}

The authors would like to thank J. Ferreira (Dept of Statistics and Modelling, National Institute for Public Health and the Environment, Bilthoven, the Netherlands) for statistical advice and T. Rygiel (Dept Immunology, University Medical Center Utrecht, Utrecht, the Netherlands) for invaluable laboratory support and B. Nieuwenstein, (Dept of Medical Technology, University Medical Center Utrecht, Utrecht, the Netherlands) for excellent technical assistance. 


\section{REFERENCES}

1 Falsey AR, Hennessey PA, Formica MA, et al. Respiratory syncytial virus infection in elderly and high-risk adults. $N$ Engl J Med 2005; 352: 1749-1759.

2 Tregoning JS, Schwarze J. Respiratory viral infections in infants: causes, clinical symptoms, virology, and immunology. Clin Microbiol Rev 2010; 23: 74-98.

3 American Academy of Pediatrics Subcommittee on Diagnosis and Management of Bronchiolitis. Diagnosis and management of bronchiolitis. Pediatrics 2006; 118: 1774-1793.

4 Behrendt CE, Decker MD, Burch DJ, et al. International variation in the management of infants hospitalized with respiratory syncytial virus. International RSV Study Group. Eur J Pediatr 1998; 157: 215-220.

5 Simoes EA. Respiratory syncytial virus infection. Lancet 1999; 354 847-852.

6 McNamara PS, Ritson P, Selby A, et al. Bronchoalveolar lavage cellularity in infants with severe respiratory syncytial virus bronchiolitis. Arch Dis Child 2003; 88: 922-926.

7 Lukens MV, van de Pol AC, Coenjaerts FE, et al. A systemic neutrophil response precedes robust $\mathrm{CD} 8(+)$ T-cell activation during natural respiratory syncytial virus infection in infants. J Virol 2010; 84: 2374-2383.

8 Janssen R, Pennings J, Hodemaekers H, et al. Host transcription profiles upon primary respiratory syncytial virus infection. J Virol 2007; 81: 5958-5967.

9 Dreyfuss D, Saumon G. Ventilator-induced lung injury: lessons from experimental studies. Am J Respir Crit Care Med 1998; 157: 294-323.

10 Pinhu L, Whitehead T, Evans T, et al. Ventilator-associated lung injury. Lancet 2003; 361: 332-340.

11 Laffey JG, Honan D, Hopkins N, et al. Hypercapnic acidosis attenuates endotoxin-induced acute lung injury. Am J Respir Crit Care Med 2004; 169: 46-56.

12 Shibata K, Cregg N, Engelberts D, et al. Hypercapnic acidosis may attenuate acute lung injury by inhibition of endogenous xanthine oxidase. Am J Respir Crit Care Med 1998; 158: 1578-1584.

13 Sinclair SE, Kregenow DA, Lamm WJ, et al. Hypercapnic acidosis is protective in an in vivo model of ventilator-induced lung injury. Am J Respir Crit Care Med 2002; 166: 403-408.

14 Coakley RJ, Taggart $\mathrm{C}$, Greene $\mathrm{C}$, et al. Ambient $\mathrm{PCO}_{2}$ modulates intracellular $\mathrm{pH}$, intracellular oxidant generation, and interleukin-8 secretion in human neutrophils. J Leukoc Biol 2002; 71: 603-610.

15 West MA, Hackam DJ, Baker J, et al. Mechanism of decreased in vitro murine macrophage cytokine release after exposure to carbon dioxide: relevance to laparoscopic surgery. Ann Surg 1997; 226: 179-190

16 Curley G, Contreras MM, Nichol AD, et al. Hypercapnia and acidosis in sepsis: a double-edged sword? Anesthesiology 2010; 112: $462-472$.

17 Schuurhof A, Bont L, Pennings JL, et al. Gene expression differences in lungs of mice during secondary immune responses to respiratory syncytial virus infection. J Virol 2010; 84: 9584-9594.
18 Raychaudhuri S, Stuart JM, Altman RB. Principal components analysis to summarize microarray experiments: application to sporulation time series. Pac Symp Biocomput 2000; 455-466.

19 Ricard JD, Dreyfuss D, Saumon G. Production of inflammatory cytokines in ventilator-induced lung injury: a reappraisal. Am J Respir Crit Care Med 2001; 163: 1176-1180.

20 Gharib SA, Liles WC, Klaff LS, et al. Noninjurious mechanical ventilation activates a proinflammatory transcriptional program in the lung. Physiol Genomics 2009; 37: 239-248.

21 Papaiahgari S, Yerrapureddy A, Reddy SR, et al. Genetic and pharmacologic evidence links oxidative stress to ventilatorinduced lung injury in mice. Am J Respir Crit Care Med 2007; 176: 1222-1235.

22 Altemeier WA, Matute-Bello G, Gharib SA, et al. Modulation of lipopolysaccharide-induced gene transcription and promotion of lung injury by mechanical ventilation. I Immunol 2005; 175: 3369-3376.

23 Jafri HS, Chavez-Bueno S, Mejias A, et al. Respiratory syncytial virus induces pneumonia, cytokine response, airway obstruction, and chronic inflammatory infiltrates associated with long-term airway hyperresponsiveness in mice. J Infect Dis 2004; 189: 1856-1865.

24 Openshaw PJ. Immunity and immunopathology to respiratory syncytial virus. The mouse model. Am J Respir Crit Care Med 1995; 152: S59-S62.

25 Peebles RS Jr, Graham BS. Pathogenesis of respiratory syncytial virus infection in the murine model. Proc Am Thorac Soc 2005; 2 110-115.

26 Bem RA, van Woensel JB, Bos AP, et al. Mechanical ventilation enhances lung inflammation and caspase activity in a model of mouse pneumovirus infection. Am J Physiol Lung Cell Mol Physiol 2009; 296: L46-L56.

27 Zosky GR, Cannizzaro V, Hantos Z, et al. Protective mechanical ventilation does not exacerbate lung function impairment or lung inflammation following influenza A infection. J Appl Physiol 2009; 107: 1472-1478.

28 Moscinski LC, Hill B. Molecular cloning of a novel myeloid granule protein. J Cell Biochem 1995; 59: 431-442.

29 Catalano RD, Lannagan TR, Gorowiec M, et al. Prokineticins: novel mediators of inflammatory and contractile pathways at parturition? Mol Hum Reprod 2010; 16: 311-319.

30 Leclerc F, Scalfaro P, Noizet $\mathrm{O}$, et al. Mechanical ventilatory support in infants with respiratory syncytial virus infection. Pediatr Crit Care Med 2001; 2: 197-204.

31 Domachowske JB, Bonville CA, Rosenberg HF. Animal models for studying respiratory syncytial virus infection and its long term effects on lung function. Pediatr Infect Dis J 2004; 23: S228-S234.

32 Rosenberg HF, Domachowske JB. Pneumonia virus of mice: severe respiratory infection in a natural host. Immunol Lett 2008; 118: 6-12.

33 van Schaik SM, Enhorning G, Vargas I, et al. Respiratory syncytial virus affects pulmonary function in BALB/c mice. J Infect Dis 1998; 177: $269-276$ 